RUHN-00-01

\title{
An alternative to domain wall fermions
}

\author{
Rajamani Narayanan \\ American Physical Society, One Research Road, Ridge, NY 11961 \\ rajamani@bnl.gov \\ Herbert Neuberger \\ Department of Physics and Astronomy, Rutgers University, Piscataway, NJ 08855-0849 \\ neuberg@physics.rutgers.edu
}

\begin{abstract}
We define a sparse hermitian lattice Dirac matrix, $H$, coupling $2 n+1$ Dirac fermions. When $2 n$ fermions are integrated out the induced action for the last fermion is a rational approximation to the hermitian overlap Dirac operator. We provide rigorous bounds on the condition number of $H$ and compare them to bounds for the higher dimensional Dirac operator of domain wall fermions. Our main conclusion is that overlap fermions should be taken seriously as a practical alternative to domain wall fermions in the context of numerical QCD.
\end{abstract}




\section{INTRODUCTION}

A major embarrassment of lattice field theory in the context of QCD has disappeared: we now have a way to preserve chiral symmetry on the lattice [1 [3], and the all important theoretical understanding of "soft" physics consequences of chiral symmetry at the Lagrangian level can be taken over from the continuum to the lattice. Naturally, one is eager to exploit this development in numerical QCD, and at the moment there are two ways that have been explored: One is the so called domain wall fermion approach [1, 3, 14, and the other is based on implementing [5] a rational approximation to the sign function of the overlap Dirac operator [6]. The relation between the two is elucidated in [7]. One conclusion from [7] could be that with computers immensely more powerful than the ones we have at present it wouldn't matter which approach one uses for numerical QCD. In practice, the two methods are quite different, and it is important to assess their relative strengths and weaknesses.

To compare methods one needs to separate the quenched case from the dynamical case: In a dynamical hybrid Monte Carlo simulation one needs to invert the Dirac operator often, at each step of the so called "trajectory". The inversions do not generate propagators used in computing physical observables, except when the trajectory is completed and the change in gauge fields accepted. In a quenched simulation one extracts physics results from each set of fermion propagators. Thus, the comparison of overall fractional costs for inversions works out differently in the quenched and in the dynamical case.

It seems that the overlap approach has some advantage in the quenched case: it has been possible to do simulations at very low quark masses [8], lower than what was achieved with domain wall fermions [9]. To be sure, a systematic and complete comparison has not been carried out, so this is more an impression than a hard fact. The reason for the difference is that with the overlap it has been possible to better handle the cases where the Hermitian Wilson Dirac operator, $H_{W}$, has eigenvalues very close to zero. Numerical investigation has shown that there is a finite density of eigenvalues very close to zero at typical lattice couplings used in lattice simulations [10]. While this is a problem for both domain walls [7] and overlap, in the overlap case one can exploit the simple local structure of $H_{W}$ and project out the troublesome states [11]. This is expensive, but needs to be done only once per gauge field configuration and will help in the calculation of all propagators at this gauge field. Thus, the extra expense is amortized in the quenched case. A similar projection method in the case of domain wall fermions might make both methods equal. Until very recently there existed no practical implementation of a projection technique in the domain wall context, but this is changing as we write [12].

At the dynamical level it always was felt that domain wall fermions were superior because the action was quite standard in form and one needed only one conjugate gradient inversion, rather than the two nested ones required by the rational overlap [5, 11]. Here again, this is more an impression than a hard fact because one ought to take into account condition numbers, matrix sizes and the possibility to use projectors. But, superficially at least, it is hard to ignore the advantage of domain wall fermions with a straightforward action and a relatively well tested algorithm.

The latter distinction between the implementation of domain wall fermions and rational overlap fermions could be eliminated however [13] by undoing what the overlap does relative to domain wall fermions. One reintroduces extra fields which interact quadratically by a 
sparse hermitian matrix $H$. The main requirement of $H$ is the following: There exists one massless field, $\psi$, such that integrating out all the other fields produces for $\psi$ a specific effective action $S_{\text {eff }}$. $S_{\text {eff }}=-\bar{\psi} H_{o}^{n} \psi$ and the approximate hermitian overlap Dirac operator is given by $H_{o}^{n}=\frac{1}{2}\left(\gamma_{5}+\epsilon_{n}\left(H_{W}\right)\right)$ where the function $\epsilon_{n}(x)$ is a numerically accurate rational approximation to the sign function $\operatorname{sign}(x)$ for $x$ in the spectrum of $H_{W}$. One can easily add an explicit Dirac mass term for the field $\psi$.

$H$ has to fulfill additional requirements: It has to have a condition number that is not significantly worse than that of the five dimensional Dirac operator used for domain wall fermions, $D$. In particular, the condition number should not diverge as a function of the number of extra fields, $2 n$. The cost of acting with $H$ should grow no more than linearly with $n$, so long as the truncation of the sign function converges as $e^{-c n}$. Furthermore, $H$ should depend structurally only on $H_{W}$ as one entity. A dependence on $H_{W}$ as one object ensures that if a better version of $H_{W}$ is eventually found, it would be trivial to change $H$, replacing the old $H_{W}$ with the new one. Also, the implementation of the projection technique simply involves a replacement of $H_{W}$ as a whole. In particular, taking derivatives with respect to the gauge fields would be simple, making the computation of the "force" in hybrid Monte Carlo relatively easy. Although the action for domain wall fermions also has a simple dependence on the gauge fields, the dependence on the length of the extra direction is controlled by the complicated transfer matrix $T_{W}$ [7]. The dependence on $n$ is simpler in the overlap case, as it comes in only through $\epsilon_{n}\left(H_{W}\right)$, and $H_{W}$ is a sparse matrix, unlike $T_{W}$.

Previous proposals for $H$ had some numerical entries that were large and grew with $n$. This implied that the norm of $H,\|H\|$, grew and made the condition number $\kappa(H)=$ $\|H\|\left\|H^{-1}\right\|$ grow too. Our choice for matrix norms is quite standard: $\|X\|=\sqrt{\lambda_{\max }\left(X^{\dagger} X\right)}$, with $\lambda_{\max }\left(X^{\dagger} X\right)$ being the largest eigenvalue of $X^{\dagger} X$. The main objective of this paper is to present a new version of $H$ which satisfies the above requirements and also has a reasonable condition number.

In the next section we shall construct $H$. We proceed with the derivation of rigorous upper bounds on $\|H\|$ and on $\left\|H^{-1}\right\|$. Rigorous bounds are nice, but there always is a question whether they are saturated. This will be discussed. We shall also ask the opposite question: what is the best one can hope for, regarding $\kappa(H)$ ? To answer that we shall derive a lower bound for $\left\|H^{-1}\right\|$. This is all we need because, in practice, we expect the upper bound on $\|H\|$ to be typically almost saturated. We proceed to address the question how our results compare to what is known about the domain wall fermion operator $D$. Since not much seems to be known, we derive some exact results for $D$ too, but also leave more work for the future. We then briefly discuss projection techniques for both overlap and domain wall fermions.

We conclude the paper with a discussion of other advantages we envisage our proposal to have over domain wall fermions, beyond better chirality properties. We compare the number of $H_{W}$ operations needed to perform the inversion of the fermionic operator. We find this number numerically for three cases: domain wall fermions, a method using the operator $H$ proposed in this paper, and a method using a direct computation of the sign function represented by the same rational approximation as implemented by $H$. The comparison is carried out for the two dimensional Schwinger model. We hope to convince the reader that 
the overlap alternatives merit serious numerical testing in the context of QCD, something we are not fully geared up to do efficiently by ourselves.

\section{CONSTRUCTION OF $H$}

The kernels of the quadratic fermionic actions we shall work with will be hermitian matrices in most cases. Let $\psi$ be the light Dirac field representing a light quark. We wish to end up with an action

$$
S_{\text {eff }}(\psi)=-\bar{\psi}\left[\frac{1+\mu}{2} \gamma_{5}+\frac{1-\mu}{2} \varepsilon_{n}\left(H_{W}\right)\right] \psi .
$$

The bare quark mass $\mu$ [7] is restricted by $|\mu|<1$ for physical reasons.

The matrix $H_{W}$ has the standard Wilson form, but can be easily replaced by a more elaborate construction. The function $\varepsilon_{n}(x)$ is an approximation to $\operatorname{sign}(x)$ :

$$
\varepsilon_{n}\left(H_{W}\right)=\frac{1}{n} \sum_{s=1}^{n} \frac{1}{c_{s}^{2} H_{W}+\frac{s_{s}^{2}}{H_{W}}}
$$

with

$$
c_{s}=\cos \theta_{s}, \quad s_{s}=\sin \theta_{s}, \quad \theta_{s}=\frac{\pi}{2 n}\left(s-\frac{1}{2}\right), s=1,2, \ldots, n
$$

This rational approximation can be replaced by others: One can replace the $c_{s}^{2}$ and $s_{s}$ quantities with other real numbers of either sign; to change the overall sign of a contribution at some $s$ one simply switches the sign of $H_{W}$ at that $s$.

For each $s$ we introduce a new field $\chi_{s}$. The $\chi_{s}$ fields are decoupled from each other and enter quadratically in the action:

$$
\bar{\chi}_{s}\left[c_{s}^{2} H_{W}+\frac{s_{s}^{2}}{H_{W}}\right] \chi_{s} .
$$

In addition, they couple to the $\psi$ field by

$$
\sqrt{\frac{1-\mu}{2 n}} \sum_{s}\left(\bar{\psi} \chi_{s}+\bar{\chi}_{s} \psi\right) .
$$

To get the right effective action for $\psi$, there also is a quadratic term in $\psi$ :

$$
-\frac{1+\mu}{2} \bar{\psi} \gamma_{5} \psi
$$

To make the action local we should eliminate the inverse of $H_{W}$ from the $\chi$-action. To this end we introduce $n$ more Dirac fields, $\phi_{s}$, also Grassmann, and change the $\chi$ self interaction to:

$$
\bar{\chi}_{s}\left(c_{s}^{2} H_{W}\right) \chi_{s}+s_{s}\left(\bar{\chi}_{s} \phi_{s}+\bar{\phi}_{s} \chi_{s}\right)-\bar{\phi}_{s}\left(H_{W}\right) \phi_{s}
$$

Introduce the combined field $\bar{\Psi}=\left(\bar{\psi}, \bar{\chi}_{1}, \bar{\phi}_{1}, \ldots, \bar{\chi}_{n}, \bar{\phi}_{n}\right)$. The action is 


$$
S=\bar{\Psi} H \Psi
$$

with

$$
H=\left(\begin{array}{cccccccc}
-\frac{1+\mu}{2} \gamma_{5} & \sqrt{\frac{1-\mu}{2 n}} & 0 & \sqrt{\frac{1-\mu}{2 n}} & 0 & \ldots & \sqrt{\frac{1-\mu}{2 n}} & 0 \\
\sqrt{\frac{1-\mu}{2 n}} & c_{1}^{2} H_{W} & s_{1} & 0 & 0 & \ldots & 0 & 0 \\
0 & s_{1} & -H_{W} & 0 & 0 & \ldots & 0 & 0 \\
\sqrt{\frac{1-\mu}{2 n}} & 0 & 0 & c_{2}^{2} H_{W} & s_{2} & \ldots & 0 & 0 \\
0 & 0 & 0 & s_{2} & -H_{W} & \ldots & 0 & 0 \\
\vdots & \vdots & \vdots & \vdots & & \vdots & 0 & 0 \\
\sqrt{\frac{1-\mu}{2 n}} & 0 & 0 & 0 & 0 & \ldots & c_{n}^{2} H_{W} & s_{n} \\
0 & 0 & 0 & 0 & 0 & \ldots & s_{n} & -H_{W}
\end{array}\right)
$$

Our new extended overlap model is based on the following identity:

$$
\int d \bar{\Psi} d \Psi e^{\bar{\Psi} H \Psi}=\left[\prod_{s=1}^{n} \operatorname{det}\left(c_{s}^{2} H_{W}^{2}+s_{s}^{2}\right)\right] \int d \bar{\psi} d \psi e^{-\bar{\psi}\left(\frac{1+\mu}{2} \gamma_{5}+\frac{1-\mu}{2 n} \sum_{s=1}^{n} \frac{1}{c_{s}^{2} H_{W}+\frac{s_{s}^{2}}{H_{W}}}\right) \psi}
$$

The prefactor can be canceled by adding pseudofermions, which will be decoupled in the $s$ index.

At $n=\infty$ we can take a continuum limit, with

$$
d \theta=\frac{\pi}{2 n} d s, \quad 0<\theta<\frac{\pi}{2}, \quad \chi_{s}=\frac{1}{\sqrt{n}} \chi(\theta), \quad \phi_{s}=\frac{1}{\sqrt{n}} \phi(\theta) .
$$

The effective action then becomes:

$$
\begin{aligned}
S= & -\frac{1+\mu}{2} \bar{\psi} \gamma_{5} \psi+\frac{2}{\pi} \sqrt{\frac{1-\mu}{2}}\left[\bar{\psi} \int d \theta \chi(\theta)+\int d \theta \bar{\chi}(\theta) \psi\right] \\
& +\frac{2}{\pi} \int d \theta\left[\cos ^{2} \theta \bar{\chi}(\theta) H_{W} \chi(\theta)-\bar{\phi}(\theta) H_{W} \phi(\theta)+\sin \theta \bar{\chi}(\theta) \phi(\theta)+\sin \theta \bar{\phi}(\theta) \chi(\theta)\right] .
\end{aligned}
$$

The following identity (which holds as long as $\left\|H_{W}^{-1}\right\|$ is finite) expresses the essence of our construction:

$$
\frac{2}{\pi} \int_{0}^{\frac{\pi}{2}} \frac{d \theta}{H_{W} \cos ^{2} \theta+\frac{\sin ^{2} \theta}{H_{W}}}=\frac{2}{\pi} \int_{0}^{\infty} \frac{d t}{t} \frac{1}{\frac{H_{W}}{t}+\frac{t}{H_{W}}}=\operatorname{sign}\left(H_{W}\right) .
$$

In terms of $\theta$ the action in the extra dimension involves no derivatives. In a rough sense the $\theta$ variable corresponds to a "fifth" momentum (for a four dimensional physics application). The physical degrees of freedom, $\psi$, couple only to an average field. These two features indicate that one might be able to use some sort of multi-grid or hierarchical techniques to reduce the $\mathcal{O}(n)$ computational cost per inversion of $H$ to a logarithmic dependence on $n$. Other integral representations of the sign function, or other approximation to the integrals above, will produce more variations on the same basic idea, but with possibly different computational properties. 
The strategy for finding bounds on the spectrum of $H$ is based on an exact formula for the determinant of $H-z$. This determinant is obtained by adding $-z \bar{\Psi} \Psi$ to the action and doing the gaussian integral.

$$
\operatorname{det}(H-z)=\left\{\prod_{s=1}^{n} \operatorname{det}\left[\left(c_{s}^{2} H_{W}-z\right)\left(H_{W}+z\right)+s_{s}^{2}\right]\right\} \operatorname{det}\left[\frac{1+\mu}{2} \gamma_{5}+z+\frac{1-\mu}{2} f_{n}\left(H_{W}, z\right)\right]
$$

Here,

$$
f_{n}\left(H_{W}, z\right)=\frac{1}{n} \sum_{s=1}^{n} \frac{1}{c_{s}^{2} H_{W}+\frac{s_{s}^{2}}{H_{W}+z}-z} .
$$

Eigenvalues of $H$ are roots of the equation $\operatorname{det}(H-z)=0$. All the roots come from roots of the last factor. (Roots of the factors in the product over $s$ are canceled by poles in the last factor. So, the spectrum of $H$ is determined by the last factor.)

Introducing

$$
S_{n}(a, b)=\frac{1}{n} \sum_{s=1}^{n} \frac{1}{a c_{s}^{2}+b s_{s}^{2}}
$$

we see that

$$
f_{n}\left(H_{W}, z\right)=S_{n}\left(H_{W}-z, \frac{1}{H_{W}+z}-z\right) .
$$

$S_{n}(a, b)$ is a ratio of polynomials in $a, b$ :

$$
S_{n}(a, b)=\frac{P_{n}(a, b)}{Q_{n}(a, b)}, \quad P_{n}(a, b)=\sum_{s=1}^{n}\left(\begin{array}{c}
2 n \\
2 s-1
\end{array}\right) b^{n-s} a^{s-1}, \quad Q_{n}(a, b)=\sum_{s=0}^{n}\left(\begin{array}{c}
2 n \\
2 s
\end{array}\right) b^{n-s} a^{s} .
$$

Extracting $\left(H_{W}+z\right)^{-n}$ from numerator and denominator, we see that $S(a, b)$ is also a ratio of polynomials in $H_{W}$. For real $a$ and $b$ we can write simple closed formulae: If $a b>0$ we have

$$
S_{n}(a, b)=\frac{\operatorname{sign}(b)}{\sqrt{a b}} \tanh (n \omega), \quad \omega=\log (|1+\sqrt{a / b}| /|1-\sqrt{a / b}|) .
$$

If $a b<0$,

$$
S_{n}(a, b)=\frac{\operatorname{sign}(b)}{\sqrt{-a b}} \tan (n \omega), \quad 0 \leq \omega \leq \frac{\pi}{2}, \quad e^{i \omega}=(1+i \sqrt{-a / b}) /(1-i \sqrt{-a / b}) .
$$

These formulae make the $n$-dependence explicit.

The product $a b$ clearly plays a central role:

$$
a b=1+z g\left(H_{W}, z\right), \quad g\left(H_{W}, z\right)=z-H_{W}-\frac{2}{H_{W}+z} .
$$

Our bounds are based on the observation that for $a b>0$ we have an $n$-independent bound:

$$
\left|f_{n}\left(H_{W}, z\right)\right|=\left|S_{n}(a, b)\right| \leq \frac{1}{\sqrt{a b}} .
$$

If we allowed $a b<0$, we would have had little control over $S_{n}(a, b)$ because of the tangent function. 


\section{UPPER BOUND ON $\|H\|$}

For very large $|z| a b$ is large and positive, making $z+\frac{1-\mu}{2} f_{n}\left(H_{W}, z\right)$ large in absolute value. It is clear that $\operatorname{det}(H-z)$ cannot vanish then. To find an upper bound for $\|H\|$ we look for the smallest $|z|$ values for which the above is still true, as we decrease $|z|$ from infinity.

Theorem I: For any hermitian bounded $H_{W}$ we have

$$
\|H\| \leq \sqrt{\left\|H_{W}\right\|^{2}+2} .
$$

Proof of Theorem I: Pick a real number $z$,

$$
|z| \geq \sqrt{\left\|H_{W}\right\|^{2}+2}
$$

We shall prove, by contradiction, that this $\operatorname{implies} \operatorname{det}(H-z) \neq 0$. All we need to show is that the hermitian operator $\frac{1+\mu}{2} \gamma_{5}+z+\frac{1-\mu}{2} f_{n}\left(H_{W}, z\right)$ has no zero eigenvectors. We assume that there exists such a normalized eigenvector $\psi_{0}$. $\psi_{0}$ obeys:

$$
\frac{1+\mu}{2} \gamma_{5} \psi_{0}=-\left[z+\frac{1-\mu}{2} f_{n}\left(H_{W}, z\right)\right] \psi_{0}
$$

Taking norms we get

$$
\frac{1+\mu}{2}=\sqrt{\psi_{0}^{\dagger}\left[z+\frac{1-\mu}{2} f_{n}\left(H_{W}, z\right)\right]^{2} \psi_{0}} .
$$

Let $h$ be an arbitrary eigenvalue of $H_{W}$; we know that

$$
|z| \geq \sqrt{h^{2}+2}
$$

Simple analysis of the function $z g(h, z)$ implies

$$
z g(h, z) \geq 0 \text {. }
$$

As a consequence:

$$
\left|f_{n}(h, z)\right| \leq 1
$$

Hence, every eigenvalue $\lambda$ of $z+\frac{1-\mu}{2} f_{n}\left(H_{W}, z\right)$ obeys

$$
|\lambda| \geq|z|-\frac{1-\mu}{2}
$$

(Recall that $|\mu| \leq 1$.) By the variational principle, the right hand side of equation (25) obeys the same inequality. Hence,

$$
\frac{1+\mu}{2} \geq|z|-\frac{1-\mu}{2}
$$

which implies $|z| \leq 1$ in contradiction to our initial assumption about $z$ 
This establishes the upper bound of Theorem I. Note that it is $\mu$ and $n$ independent. Corollary: Let $H_{W}$ be the hermitian Wilson Dirac operator in even $d$ dimensions, and $H$ defined as above, with the standard replacement of the four dimensional $\gamma_{5}$. Let the mass parameter $m$ in $H_{W}$ be restricted by $m>-2$. Then

$$
\|H\| \leq \sqrt{2+(2 d+m)^{2}} .
$$

This is a direct consequence of the known upper bound on $\left\|H_{W}\right\|$

We expect this bound to be quite close to optimal.

This proves that the main difficulty faced by previous proposals to implement the overlap Dirac operator by adding extra fields has been completely eliminated. The problem with the previous proposals was that one could not control $\|H\|$. Moreover, our bound is quite stringent numerically, even smaller than a typical bound on a Wilson Dirac operator in $2 d+1$ dimensions [14]. This is a plausible comparison, because one could thing about $s$ as indexing an extra dimension. At any rate, for $d=4$ and $m=-1.8$ one would have

$$
\|H\| \leq 6.4
$$

which is very reasonable.

We ran some tests in two dimensions with $m=-1$ and found the bound of 3.3 to be typically almost saturated for $U(1)$ gauge configurations generated with a Wilson action at $\beta=2$. Moreover, with a trivial gauge background one can explicitly check that one can get quite close numerically to the upper bound and so it is impossible to find a gauge background independent bound which is significantly better.

\section{UPPER BOUND ON $\left\|H^{-1}\right\|$}

The immediate question we need to address now is what happens to the low eigenvalues of $H$. Having established that $H$ behaves more or less as a usual fermionic lattice operator at high eigenvalues, numerical problems can only come from a large $\left\|H^{-1}\right\|$.

The basic strategy to get the bound is similar to the one used above. First take $z=0$ and $n=\infty$. Assume that $H_{W}$ has no zero eigenvalue. Then, $a b=1$ and $\left\|f_{\infty}\left(H_{W}, 0\right)\right\|=1$. As a result, $\frac{1+\mu}{2} \gamma_{5}+\frac{1-\mu}{2} f_{\infty}\left(H_{W}, 0\right)$ cannot have zero eigenvalues if $\mu \neq 0$. We therefore look now for a neighborhood of $z=0$ where, if $\mu>0$ and $H_{W}$ has no zero mode, we have

$$
\frac{1+\mu}{2}>\left\|z+\frac{1-\mu}{2} f_{n}\left(H_{W}, z\right)\right\|
$$

If this is true, $\frac{1+\mu}{2} \gamma_{5}+z+\frac{1-\mu}{2} f_{n}\left(H_{W}, z\right)$ cannot have a zero mode. Indeed, if there were such a zero mode, $\psi_{0}$, we would have a contradiction, since then

$$
\left(\frac{1+\mu}{2}\right)^{2} \psi_{0}^{\dagger} \psi_{0}=\psi_{0}^{\dagger}\left[z+\frac{1-\mu}{2} f_{n}\left(H_{W}, z\right)\right]^{2} \psi_{0}
$$

in violation of the variational principle for the maximal eigenvalue of $\left[z+\frac{1-\mu}{2} f_{n}\left(H_{W}, z\right)\right]^{2}$. 
The range $0<\mu<1$ covers all possible positive quark masses. The case $-1<\mu<0$ describes quarks with a negative mass. This certainly is not without interest, but the analysis becomes more complicated and unnecessary for our purposes here. So, we simply restrict ourselves to the range $0<\mu<1$.

Let $h$ be an eigenvalue of $H_{W}$ :

$$
\frac{1}{\left\|H_{W}^{-1}\right\|} \leq|h| \leq\left\|H_{W}\right\| .
$$

We define $z_{n}(h, \mu)$ as the smallest positive solution to the following equation:

$$
\frac{1+\mu}{2}=\left|z_{n}(h, \mu)+\frac{1-\mu}{2} f_{n}\left(h, z_{n}(h, \mu)\right)\right| .
$$

Since $f_{n}(-h,-z)=-f_{n}(h, z)$, we have:

$$
z_{n}(h, \mu)=-z_{n}(-h, \mu)
$$

$h$ is restricted to ranges that are symmetric about zero. Therefore, the inequality

$$
\frac{1+\mu}{2}>\left|z+\frac{1-\mu}{2} f_{n}(h, z)\right|
$$

can be guaranteed by restricting $z$ to a range symmetric about zero:

$$
|z|<u_{n}\left(H_{W}, \mu\right)
$$

Here, the numerical bound $u_{n}\left(H_{W}, \mu\right)$ is given by

$$
u_{n}\left(H_{W}, \mu\right)=\min _{\frac{1}{\left\|H_{W}^{-1}\right\|} \leq|h| \leq\left\|H_{W}\right\|} z_{n}(h, \mu) .
$$

We have therefore proven the following bound:

Theorem II: For $0 \leq \mu<1$ and arbitrary nonsingular hermitian $H_{W}$, we have:

$$
\frac{1}{\left\|H^{-1}\right\|} \geq u_{n}\left(H_{W}, \mu\right) \text {. }
$$

$u_{n}\left(H_{W}, \mu\right)$ is determined by equations (36) and (40)

$z_{n}(h, \mu)$ can be found numerically for the ranges of $h$ and $\mu$ of interest in practice. To find a bound analytically is cumbersome because $u_{n}\left(H_{W}, \mu\right)$ will be controlled by either an $h$ corresponding to a maximal (in absolute magnitude) eigenvalue of $H_{W}$ or to a minimal one. Some analysis shows that there is a constant of order unity (dependent on the dimension $d$ and the $m$ parameter in $\left.H_{W}\right), C$, and that if we restrict $H_{W}$ by

$$
\left\|H_{W}^{-1}\right\| \geq C
$$

the minimum over $h$ we need in order to get $u_{n}\left(H_{W}, \mu\right)$ is attained at

$$
h=h_{\min }=\frac{1}{\left\|H^{-1}\right\|} .
$$


Thus, the entire dependence of $u_{n}\left(H_{W}, \mu\right)$ on $H_{W}$ comes in through the value of the lowest eigenvalue of $H_{W}^{2}$. Gauge configurations for which $\left\|H_{W}^{-1}\right\|<C$ are easily handled by any numerical method and the condition number in that case is not a source of concern. So, nothing is lost by assuming that the smallest eigenvalue of $H_{W}^{2}$ is smaller than $C^{-2}$; this is anyhow the case for most gauge configurations one encounters in practical QCD simulations.

In order to get some feeling for orders of magnitude we work out a simple analytical approximation for the bound on $|z|$ and represent the exact result for the bound as the product of this approximation times a correction factor. Some typical values for the cor-

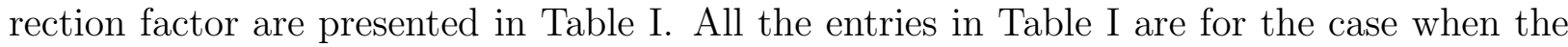
smallest eigenvalue of $H_{W}^{2}$ is smaller than $C^{-2}$.

We now take $a b>0$ because this certainly is true at $z=0$. As explained above, we can restrict our attention to positive $z$, without loosing generality. It is true now that

$$
\left\|z+\frac{1-\mu}{2} f_{n}\left(h_{\min }, z\right)\right\| \leq z+\frac{1-\mu}{2} \frac{1}{\sqrt{1+z g\left(h_{\min }, z\right)}} .
$$

So, our bound will hold if we enforce the first inequality below:

$$
\begin{aligned}
\frac{1+\mu}{2} & >z+\frac{1-\mu}{2} \frac{1}{\sqrt{1+z g\left(h_{\min }, z\right)}} \approx z+\frac{1-\mu}{2}\left[1+z\left(\frac{h_{\min }}{2}+\frac{1}{h_{\min }}\right)\right] \\
& \approx z+\frac{1-\mu}{2}+\frac{1-\mu}{2} \frac{z}{h_{\min }} \approx \frac{1-\mu}{2}+\frac{z}{2 h_{\min }} .
\end{aligned}
$$

In the above series of approximations we assumed $h_{\min }<<1$. We end up with an approximate range from which eigenvalues of $H$ are excluded:

$$
|z|<2 \mu h_{\min }
$$

Finally, we rewrite our exact result in the following form:

$$
\frac{1}{\left\|H^{-1}\right\|} \geq u_{n}\left(H_{W}, \mu\right) \equiv c_{n}\left(H_{W}, \mu\right) \frac{2 \mu}{\left\|H_{W}^{-1}\right\|}
$$

For $\left\|H_{W}^{-1}\right\| \geq C$ the $c_{n}$ prefactor depends on $H_{W}$ just through $h_{\min }$. In Table $\mathbb{\theta}$ we collected several values of $c_{n}\left(h_{\min }, \mu\right)$ to give a sense of the dependence on $n, h_{\min }$ and $\mu . c_{n}\left(H_{W}, \mu\right)$ is calculated using equations (36), (40) and (47).

\section{CONDITION NUMBER OF $H$ : WORST AND BEST CASE}

Combining our exact results we obtain an exact bound for condition numbers.

\section{Theorem III:}

$$
\kappa(H) \leq \frac{\kappa\left(H_{W}\right)}{2 \mu} \frac{\sqrt{1+\frac{2}{\left\|H_{W}\right\|^{2}}}}{c_{n}\left(H_{W}, \mu\right)}
$$




\begin{tabular}{lcccccc}
\hline \hline$h$ & $\mu$ & $n=10$ & $n=20$ & $n=50$ & $n=100$ & $n=\infty$ \\
\hline .01 & .01 & 158.9903 & 68.2685 & 15.1871 & 2.7847 & .9804 \\
.01 & .02 & 80.9208 & 34.9814 & 8.1349 & 1.8857 & .9803 \\
.01 & .04 & 41.8905 & 18.3404 & 4.6106 & 1.4366 & .9796 \\
.01 & .08 & 22.3825 & 10.0234 & 2.8506 & 1.2111 & .9759 \\
.01 & .16 & 12.6341 & 5.8657 & 1.9686 & 1.0892 & .9600 \\
.02 & .01 & 65.9303 & 22.8212 & 2.7302 & .9939 & .9616 \\
.02 & .02 & 33.7907 & 11.9821 & 1.8492 & .9779 & .9617 \\
.02 & .04 & 17.7244 & 6.5649 & 1.4094 & .9696 & .9614 \\
.02 & .08 & 9.6970 & 3.8598 & 1.1892 & .9630 & .9587 \\
.02 & .16 & 5.6891 & 2.5087 & 1.0718 & .9478 & .9451 \\
.04 & .01 & 21.7717 & 4.7348 & .9567 & .9258 & .9258 \\
.04 & .02 & 11.4341 & 2.8394 & .9417 & .9262 & .9262 \\
.04 & .04 & 6.2683 & 1.8934 & .9344 & .9266 & .9266 \\
.04 & .08 & 3.6903 & 1.4224 & .9297 & .9255 & .9255 \\
.04 & .16 & 2.4066 & 1.1852 & .9187 & .9162 & .9162 \\
.08 & .01 & 4.3730 & 1.0017 & .8606 & .8606 & .8606 \\
.08 & .02 & 2.6252 & .9322 & .8614 & .8614 & .8614 \\
.08 & .04 & 1.7535 & .8985 & .8628 & .8628 & .8628 \\
.08 & .08 & 1.3211 & .8826 & .8641 & .8641 & .8641 \\
.08 & .16 & 1.1076 & .8719 & .8612 & .8612 & .8612 \\
.16 & .01 & .8695 & .7517 & .7515 & .7515 & .7515 \\
.16 & .02 & .8118 & .7528 & .7527 & .7527 & .7527 \\
.16 & .04 & .7846 & .7549 & .7549 & .7549 & .7549 \\
.16 & .08 & .7738 & .7586 & .7586 & .7586 & .7586 \\
.16 & .16 & .7714 & .7629 & .7629 & .7629 & .7629 \\
\hline \hline
\end{tabular}

TABLE I. The entries are numerically obtained exact values for the coefficient $c_{n}(h, \mu)$ appearing in our rigorous bounds. $h^{2}$ always is the smallest eigenvalue of $H_{W}^{2}$. 
From Table [ we see that decreasing $n$ improves the condition number. This is expected because finite $n$ effects induce chirality violation even at $\mu=0$, so act somewhat as an

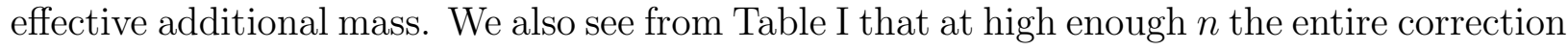
needed to make the approximate bound rigorous is of order unity. The dependence of the correction factor on $h$ and $\mu$ shows that the approximation is somewhat pessimistic.

In summary, roughly, the main message is that the condition number of $H$ cannot be worse than the product of the condition numbers for $H_{W}$ and for the effective action governing the light fermions,

$$
\kappa\left(\frac{1+\mu}{2} \gamma_{5}+\frac{1-\mu}{2} \varepsilon\left(H_{W}\right)\right) \sim \frac{1}{\mu} .
$$

It is natural to ask now: How pessimistic is the bound when viewed as an estimate for $\kappa(H)$ ? We shall answer this question in two ways: First, we shall find an upper bound to $\frac{1}{\left\|H^{-1}\right\|}$. Since the upper bound we found for $\|H\|$ is a good estimate, this would provide us with a best possible condition number. Next, we shall show that one cannot rule out a background gauge field configuration for which the lower bound on $\frac{1}{\left\|H^{-1}\right\|}$ is saturated. These two results put $\kappa(H)$ into a range. The remaining practical question is where in the range will $\kappa(H)$ typically be. The answer to this question will depend on details of the pure gauge action, and on whether we are dealing with a quenched simulation or with a dynamical one.

Upper bounds for the smallest eigenvalue of $H^{2}$ are easily obtained from the variational principle. Look at all the block diagonal elements of $H^{2}$ :

$$
H_{\psi \psi}^{2}=\frac{3+\mu^{2}}{4}, \quad H_{\chi_{s} \chi_{s}}^{2}=c_{s}^{4} H_{W}^{2}+s_{s}^{2}+\frac{1-\mu}{2 n}, \quad H_{\phi_{s} \phi_{s}}^{2}=H_{W}^{2}+s_{s}^{2} .
$$

Imagine computing the expectation value of $H^{2}$ in a state with an eigenstate of $H_{W}$ with eigenvalue $h$ in one block, and zero for all other blocks. The variational principle implies then Theorem IV.

Theorem IV:

$$
\lambda_{\min }\left(H^{2}\right) \leq \min _{h}\left\{\frac{3+\mu^{2}}{4}, \min _{s}\left(c_{s}^{4} h^{2}+s_{s}^{2}+\frac{1-\mu}{2 n}\right), \min _{s}\left(h^{2}+s_{s}^{2}\right)\right\}
$$

We assume that $h_{\text {min }} \leq \frac{1}{\sqrt{2}}$. Then, the minimum is not attained in the $\psi \psi$ block. In the other blocks, the best is to take $h=h_{\min }$. Among the $\chi_{s} \chi_{s}$ blocks and the $\phi_{s} \phi_{s}$ blocks the minimum occurs at $s=1$. Thus we find:

$$
\lambda_{\min }\left(H^{2}\right) \leq \min \left\{\left(\left(1-\sin ^{2} \frac{\pi}{4 n}\right)^{2} h_{\min }^{2}+\sin ^{2} \frac{\pi}{2 n}+\frac{1-\mu}{2 n}\right),\left(h_{\min }^{2}+\sin ^{2} \frac{\pi}{2 n}\right)\right\} .
$$

In the physical relevant cases, $\mu$ will be small enough and $n$ will be large enough to give:

$$
\frac{1}{\left\|H^{-1}\right\|} \leq \sqrt{\frac{1}{\left\|H_{W}^{-1}\right\|^{2}}+\sin ^{2} \frac{\pi}{2 n}}
$$


To get a good approximation for the sign function we need $n>>\max \left\{\left\|H_{W}\right\|, \frac{1}{\left\|H_{W}^{-1}\right\|}\right\}$, so

$$
\sqrt{\frac{1}{\left\|H_{W}^{-1}\right\|^{2}}+\sin ^{2} \frac{\pi}{2 n}} \approx \frac{1}{\left\|H_{W}^{-1}\right\|}
$$

From this we learn that, roughly, the condition number of $H$ cannot be better than the condition number of $H_{W}$. If we use a low value of $n$, for which the approximation to the sign function is bad, and hence when there are significant violations of chirality beyond the explicit mass term $\mu$, the condition number of $H$ might be better than that of $H_{W}$. This situation however probably defeats the purpose of using the new fermions instead of the older, more economical approach of fine tuning the mass term in $H_{W}$ to small effective quark mass.

On physical grounds it is obvious that there also must exist an upper bound on the lowest eigenvalue of $H^{2}$ in terms of the "bare" quark mass $\mu$. To see this we recall that the basic identity of the entire approach we are focusing on in this paper is

$$
\left(H^{-1}\right)_{\psi \psi}=\frac{1}{\frac{1+\mu}{2} \gamma_{5}+\frac{1-\mu}{2} \varepsilon_{n}\left(H_{W}\right)} .
$$

The variational principle then gives us Theorem $\mathrm{V}$.

\section{Theorem V:}

$$
\lambda_{\min }\left(H^{2}\right) \leq \lambda_{\min }\left[\frac{1+\mu}{2} \gamma_{5}+\frac{1-\mu}{2} \varepsilon_{n}\left(H_{W}\right)\right]^{2} .
$$

Proof of Theorem V: Let $\Phi=\left(\psi_{0}, 0,0, \ldots\right)$. On very general grounds, we have

$$
\frac{1}{\lambda_{\min }\left(H^{2}\right)} \geq\left\langle\Phi\left|H^{-2}\right| \Phi\right\rangle \geq\left\langle\Phi\left|H^{-1}\right| \Phi\right\rangle^{2}=\left\langle\psi_{0}\left(H^{-1}\right)_{\psi \psi} \psi_{0}\right\rangle
$$

Choosing $\psi_{0}$ as the eigenstate of $\left(\frac{1+\mu}{2} \gamma_{5}+\frac{1-\mu}{2} \varepsilon_{n}\left(H_{W}\right)\right)$ of lowest eigenvalue in absolute value establishes the inequality

Note that this inequality is a direct consequence of the existence of some light fermion in the theory described by $H$. Thus, this inequality is very general.

One cannot exclude backgrounds for which $\varepsilon_{\infty}\left(H_{W}\right)$ and $\gamma_{5}$ have a common eigenvector with the eigenvalues of opposite sign. Such eigenvectors should occur in instanton backgrounds, for example. For such a background the right hand side of the above equation, which is always bounded from below by $\mu$ (assume $\mu \geq 0$, as before) practically saturates the bound. In summary, we can say that there are gauge field backgrounds for which we know for sure that $\lambda_{\min }\left(H^{2}\right) \leq \mu^{2}$. Note that while any eigenvector of $H_{W}$ is also an eigenvector of $\varepsilon_{\infty}\left(H_{W}\right)$ the opposite is not true.

We see that one cannot hope that the condition number of $H$ would be smaller than either the condition number of $H_{W}$, or than a number of order $\frac{1}{\mu}: \kappa(H) \geq \max \left\{\kappa\left(H_{W}\right), \frac{1}{\mu}\right\}$. The upper bound on $\kappa(H)$, which we derived previously (equation (48)), said that the condition number of $H$ cannot be worse (larger) than, roughly, $\frac{\kappa\left(H_{W}\right)}{\mu}$.

The last question is whether we can imagine a gauge background for which the pessimistic (upper) bound (equation (48)) on $\kappa(H)$ is saturated. The answer is that one can. If the 
eigenstate of $H_{W}$ with eigenvalue closest to zero also is an eigenstate of $\gamma_{5}$ the worst case will be realized. In practice however this will happen rarely. The most common low eigenstates of $H_{W}^{2}$, which are the main source of difficulties in simulations, are typically non-degenerate and far from chiral (as can be checked by computing the expectation value of $\gamma_{5}$, which would be \pm 1 for a chiral state). Therefore, in simulations with typical parameters employed today, one does not expect to often realize the worst case.

It is of course important to see how these analytic considerations apply to practical simulations. As a toy model we simulated 2-dimensional QED with gauge coupling $\beta=4$ on an $8 \times 8$ lattice. The main factor governing convergence was the minimal eigenvalue in absolute value of $H$. We found that the upper bound of equation 48, when viewed as an estimate, is overly pessimistic by a factor of order 2 .

\section{DOMAIN WALL FERMIONS}

For domain wall fermions we have, in total, $n$ Dirac fermions labeled by $s$. The light fermion is not as sharply identified as before. Its left handed and right handed components reside predominantly at $s=1$ and at $s=n$. The action is $\bar{\Psi} D \Psi$, where, adopting notation from [3], we have in four dimensions

$$
D=D_{W}-P_{L}(M-1)-P_{R}(M-1)^{\dagger}, \quad P_{L}=\frac{1+\gamma_{5}}{2}, P_{R}=\frac{1-\gamma_{5}}{2} .
$$

$D_{W}$ is the Wilson Dirac operator with mass $m$ in the range $(-2,0)$, as before. $D_{W}$ is unity in $s$-space. $M-1$ and $(M-1)^{\dagger}$ have nontrivial action only in $s$-space, where they approximate first order derivatives plus a mass term coupled predominantly to the lightest fermion.

$$
M=\left(\begin{array}{ccccccc}
0 & 1 & 0 & 0 & 0 & \ldots & 0 \\
0 & 0 & 1 & 0 & 0 & \ldots & 0 \\
\vdots & \vdots & \vdots & \vdots & \vdots & \ldots & \vdots \\
0 & 0 & 0 & 0 & 0 & \ldots & 1 \\
-\mu & 0 & 0 & 0 & 0 & \ldots & 0
\end{array}\right)
$$

$D$ has a hermitian version, $H_{\text {dwf }}$. Let the hermitian matrix $S$ produce a flip in $s$-space $\left(S^{2}=1\right)$ :

$$
S=\left(\begin{array}{cccccc}
0 & 0 & 0 & \ldots & 0 & 1 \\
0 & 0 & 0 & \ldots & 1 & 0 \\
\vdots & \vdots & \vdots & \ldots & \vdots & \vdots \\
0 & 1 & 0 & \ldots & 0 & 0 \\
1 & 0 & 0 & \ldots & 0 & 0
\end{array}\right)
$$

Then, with $H_{W}=\gamma_{5} D_{W}$, we define

$$
H_{\mathrm{dwf}}=\gamma_{5} S D=S H_{W}-P_{L} M_{L}-P_{R} M_{R}
$$

leading to: 


$$
D^{\dagger} D=H_{\mathrm{dwf}}^{2}
$$

The hermitian mass operators $M_{R}=S(M-1)$ and $M_{L}=S\left(1-M^{\dagger}\right)$ are related by $S M_{R}=-M_{L} S$.

Following [7] we can derive a closed formula for $\operatorname{det}\left(D^{\dagger} D-z^{2}\right)$, but the analysis gets complicated and we are not sure that it can be completed. Therefore, we leave this issue for the future, and restrict ourselves here to just deriving an upper bound to $\lambda_{\min }\left(D^{\dagger} D\right)$. Since $\lambda_{\max }\left(D^{\dagger} D\right)$ would behave similarly to $D_{W}^{\dagger} D_{W}$ in one dimension higher, we know that $\|D\|$ will be of the order 10 for QCD [14]. Thus, an upper bound on $\frac{1}{\left\|D^{-1}\right\|}$, will effectively provide a best case for $\kappa\left(D^{\dagger} D\right)$. Our aim is to show that this best case is similar to what we have found for the model we analyzed in the previous sections.

Let $\psi_{0}$ be a normalized eigenstate of $H_{W}$ which has the smallest eigenvalue as an eigenstate of $H_{W}^{2}$. Construct a trial state in the larger space in which $D$ operates:

$$
\Psi=\frac{1}{\sqrt{n}}\left(\begin{array}{c}
\psi_{0} \\
\psi_{0} \\
\vdots \\
\psi_{0}
\end{array}\right)=S \Psi
$$

We find:

$$
H_{\mathrm{dwf}} \Psi= \pm \lambda_{\min }^{1 / 2}\left(H_{W}^{2}\right) \Psi+\frac{1+\mu}{\sqrt{n}}\left(\begin{array}{c}
-P_{R} \psi_{0} \\
0 \\
\vdots \\
0 \\
P_{L} \psi_{0}
\end{array}\right)
$$

We now take the norm

$$
\|D \Psi\|^{2}=\lambda_{\min }\left(H_{W}^{2}\right)+\frac{(1+\mu)^{2}}{n} \pm 2 \frac{1+\mu}{n} \lambda_{\min }^{1 / 2}\left(H_{W}^{2}\right) \psi_{0}^{\dagger} \gamma_{5} \psi_{0} .
$$

This leads to:

$$
\|D \Psi\|^{2} \leq \lambda_{\min }\left(H_{W}^{2}\right)+\frac{(1+\mu)^{2}}{n}+2 \frac{1+\mu}{n} \lambda_{\min }^{1 / 2}\left(H_{W}^{2}\right) .
$$

Our final bound is given by Theorem VI:

\section{Theorem VI:}

$$
\lambda_{\min }\left(D^{\dagger} D\right) \leq\left[\lambda_{\min }^{1 / 2}\left(H_{W}^{2}\right)+\frac{1+\mu}{n}\right]^{2}+(1+\mu)^{2}\left(\frac{1}{n}-\frac{1}{n^{2}}\right)
$$

For domain wall fermions to really represent massless quarks when $\mu=0$ we need [7]

$$
n \lambda_{\min }^{1 / 2}\left(H_{W}^{2}\right)>>1
$$

If we also make the stronger assumption (stronger because we loose nothing by treating only the case $\left.\lambda_{\min }\left(H_{W}^{2}\right)<1\right)$ 


$$
n \lambda_{\min }\left(H_{W}^{2}\right)>>1
$$

the right hand side of the bound in equation (67) becomes just $\lambda_{\min }\left(H_{W}^{2}\right)$ which is the same as in the previous analysis, see equations (53,54). The difference is in the finite $n$ effects: they are larger here. This could be something that works in favor of domain wall fermions. Of course, there is no guarantee that the best case, analyzed here, is a good approximation to the typical case. One can also analyze trial states with some structure in $s$-space:

$$
\Psi=\left(\begin{array}{c}
c_{1} \psi_{0} \\
c_{2} \psi_{0} \\
c_{3} \psi_{0} \\
\vdots \\
c_{n} \psi_{0}
\end{array}\right)
$$

The numerical coefficients $c_{s}$ are constrained by

$$
\sum_{s} c_{s}^{2}=1
$$

One can optimize the coefficients $c_{s}$ to attain a better bound.

Let us now argue why one cannot use rigorous methods to get a worst case condition number for domain wall fermions that is better than the worst case condition number we obtained for overlap fermions implemented by using $H$ (equation (48)).

As we learned in the previous section, the key point is that one cannot rule out, for arbitrary gauge field backgrounds, the existence of an eigenstate of $H_{W}$ with eigenvalue $h$ which also is chiral and for which $|h|$ is very small. Suppose we have such a state, call it $\psi_{0}$. This state is very special: since $\gamma_{5} H_{W}=D_{W}$, it is a simultaneous eigenstate of $H_{W}$ and $D_{W}$. We choose:

$$
\gamma_{5} \psi_{0}=\psi_{0}, \quad H_{W} \psi_{0}=D_{W} \psi_{0}=h \psi_{0} .
$$

Obviously, only a very special gauge background could accommodate a state like this, where, in addition, we want $|h|$ to be very small. Actually, we also want $h$ to be negative. If we construct a state $\Psi$ out of $\psi_{0}$ with coefficients $c_{s}$ as above, we easily see that the action of $D$ produces a state of the same structure with only the coefficients $c_{s}$ changed to $c_{s}^{\prime}$. The action on the coefficients can be immediately read off as:

$$
\left(\begin{array}{c}
c_{1}^{\prime} \\
c_{2}^{\prime} \\
\vdots \\
c_{n-1}^{\prime} \\
c_{n}^{\prime}
\end{array}\right)=[1+h-M]\left(\begin{array}{c}
c_{1} \\
c_{2} \\
\vdots \\
c_{n-1} \\
c_{n}
\end{array}\right)=\tilde{M}\left(\begin{array}{c}
c_{n} \\
c_{n-1} \\
\vdots \\
c_{2} \\
c_{1}
\end{array}\right)
$$

Here,

$$
\tilde{M}=(1+h-M) S=\left(\begin{array}{cccccc}
0 & 0 & \ldots & 0 & -1 & 1+h \\
0 & 0 & \ldots & -1 & 1+h & 0 \\
\vdots & \vdots & \ldots & \vdots & \vdots & \vdots \\
-1 & 1+h & \ldots & 0 & 0 & 0 \\
1+h & 0 & \ldots & 0 & 0 & \mu
\end{array}\right)
$$


The advantage of introducing $\tilde{M}$ is that it is hermitian and obeys

$$
[1+h-M][1+h-M]^{\dagger}=\tilde{M}^{2}
$$

We need to find the lowest eigenvalue of $\tilde{M}$ for $|h|<<1$ and to leading order in $\mu$. We are assuming, as usual, that we are close to the chiral limit where $\mu=0$ :

$$
n|h|=-n h>>1
$$

For $\mu=0$ we know that the lowest eigenvalue is practically zero [7] (it is responsible for $|\operatorname{det} \tilde{M}|=|1+h|^{n}$ being exponentially small). The eigenstate associated with this eigenvalue has the following structure: $c_{i}=N(1+h)^{i-1}$ (because $(1+h) c_{i} \approx c_{i+1}$ for a very small eigenvalue) [7]. The normalization $N$ is therefore, at $n=\infty$ [3]

$$
N=\sqrt{\frac{1}{\sum_{i=0}^{\infty}(1+h)^{2(i-1)}}}=\sqrt{-h(2+h)} .
$$

Now, first order perturbation theory gives for the smallest (in absolute value) eigenvalue of $\tilde{M}$ :

$$
\lambda_{\min }(\tilde{M}) \approx-h(2+h) \mu, \quad \sqrt{\lambda_{\min }\left(D^{\dagger} D\right)} \leq|(2+h) h| \mu .
$$

Thus, if a state of type $\psi_{0}$ exists, and under the additional assumption that $|h|<<1$, we obtain an approximate bound

$$
\sqrt{\frac{1}{\left\|\left(D^{\dagger} D\right)^{-1}\right\|}} \leq \frac{2 \mu}{\left\|H_{W}^{-1}\right\|} .
$$

This leads to essentially the same situation as in the overlap alternative: At the end of section $\mathrm{V}$ we concluded that one could not hope to be able to prove a better general bound on the condition number there than that of equation (48). Combining equation (79) with the known upper bound $\left\|D^{\dagger} D\right\|^{\frac{1}{2}} \leq(10+m)$ [14] (in four dimensions, with $-2<m \leq 0$ ), produces a bound similar to equation (48). One cannot expect to get a rigorous upper bound on the condition number of the Dirac operator employed for domain wall fermions that is superior to that obtained for the overlap implemented by $H$.

\section{RELATING PARAMETERS}

When comparing the overlap alternative to domain wall fermions one needs some criteria to relate the parameters in both models. In a real QCD simulation the criteria should be that the parameters be chosen so that similar physics is being described. This is not as unambiguous as it first sounds, but clearly beyond this paper. Here we suggest the following set of criteria: We need to prescribe relations between the number of extra fields in each case, the parameters $m$ in the Wilson Dirac operator in each case and the mass parameters $\mu$ in each case.

The easiest is to connect the integers $n$ in the two cases. This we do by requiring the error in realizing the sign function in both cases to be the same in the realistic case that $H_{W}^{2}$ 
has some small eigenvalues $|h|$, of order $10^{-2}$ or less. The error for domain wall fermions is of order

$$
e^{-\frac{1}{2} n_{\mathrm{dmf}}|h|}
$$

The error in the alternative proposed in this paper is of order

$$
e^{-2 n|h|}
$$

But the number of Dirac fields in the domain wall case is $n_{\mathrm{dmf}}$ while in the overlap alternative it is $n_{\text {ovp }}=2 n+1$ So, for practical values we should take:

$$
n_{\mathrm{dmf}} \approx 2 n_{\mathrm{ovp}}
$$

This ensures similar violations of chirality at $\mu=0$ for the gauge backgrounds where these violations are most significant. This match of the number of extra fields works in favor of the overlap alternative.

To match the parameters $m$ in both cases we focus on the problematic gauge backgrounds for which one would need very large numbers of extra fields to reproduce the sign function correctly (or else, use projection techniques). We recall that if the parameter $m$ is the same in the domain wall and overlap contexts the logarithm of the transfer matrix of domain wall fermions and the hermitian Wilson Dirac operator of the overlap both acquire eigenvalues very close to zero in the same (bad) gauge configurations [15].

What is left is to match the parameters $\mu$. This is more difficult to do in a gauge field independent way: Let us adopt the criterion that we want the quark propagator masses to be the same in both cases, for the same gauge background, and infinite number of extra fields in both cases. The parameter $m$ has already been chosen to be the same. Denoting by $u$ the fourth root of the plaquette variable [16], we find, in $d$ dimensions, the following "mean-field improved" estimates for the quark masses in each case:

For domain wall fermions, assuming $0<\mu<<1$, we get:

$$
m_{\text {phys }}^{\mathrm{dwf}}=-\frac{[m+d(1-u)][2+m+d(1-u)]}{u} \mu .
$$

For the overlap fermions we get [17]:

$$
m_{\text {phys }}^{\text {overlap }}=\frac{2 \mu}{1-\mu} \frac{|m+d(1-u)|}{u} .
$$

Tree level perturbation theory is obtained by setting $u=1$. Above, we assume that $-2<$ $m<0$. Setting the two physical masses equal to each other we obtain for small $\mu$

$$
\mu_{\text {overlap }}=\mu_{\text {dwf }}\left[1+\frac{m+d(1-u)}{2}\right] .
$$

In a QCD simulation one may take $u \approx .875, m \approx-1.8$, giving, roughly, $\mu_{\text {overlap }} \approx .35 \mu_{\text {dwf }}$. This might indicate an advantage to domain wall fermions, because the slow-down on inversion of $H$ or $D$ as a result of a small $\mu$ might be roughly the same for equal $\mu$ parameters. In our simulations of the Schwinger model $u$ is closer to unity, $u \approx .95$ 


\section{PROJECTION TECHNIQUE}

In practice, most of the numerical problems have to do with the presence of small eigenvalues to $H_{W}^{2}$ for typical gauge backgrounds. In the older applications of the overlap one used a projection method to deal with this difficulty. The projection method trivially extends to the overlap alternative presented here. Suppose we have several low states $\psi_{a}, H_{W} \psi_{a}=h_{a} \psi_{a}$ with so small $\left|h_{a}\right|$ values that the needed $n$ 's to handle these are too large. Define the orthogonal set of projectors

$$
P_{a}=\psi_{a} \psi_{a}^{\dagger}, \quad \sum_{a} P_{a}=\mathcal{P}
$$

Redefine $H_{W}$ in the overlap alternative by:

$$
H_{W} \rightarrow H_{W}^{P}=(1-\mathcal{P}) H_{W}(1-\mathcal{P})+\sum_{a} \operatorname{sign}\left(h_{a}\right) P_{a}
$$

This does not change the effective $\psi$ action at $n=\infty$; no new questions about locality appear as a result of this replacement. The replacement dramatically reduces the $n$ needed to get close to the $\mu=0$ chiral limit. The shifted states are now perfectly represented because for any $n$ we have $\varepsilon_{n}( \pm 1)= \pm 1$. Of course, calculating the $P_{a}$ 's and acting with $\mathcal{P}$ comes with a cost; it is expected that the cost is bearable because the number of states one needed to project out was of the order of 20 in practical simulations carried out so far in the quenched approximation using the rational approximation [11].

The introduction of projectors complicates the calculation of the force in hybrid Monte Carlo, but the complication is manageable [18]. Under an infinitesimal change of the background gauge fields we have:

$$
\delta P_{a}=\delta \psi_{a} \psi_{a}^{\dagger}+\psi_{a} \delta \psi_{a}^{\dagger}
$$

We make a phase choice so that $P_{a} \delta \psi_{a}=0\left(\psi_{a}\right.$ is normalized; the phase choice eliminates the component of $\delta \psi_{a}$ in the direction of $\psi_{a}$ and we ignore possible degeneracies for simplicity) and then obtain

$$
\delta \psi_{a}=\frac{1-P_{a}}{h_{a}-H_{W}} \delta H_{W} \psi_{a}
$$

The variation of the projector now follows from that of the state, and the phase choice we made no longer has any effect:

$$
\delta P_{a}=\frac{1-P_{a}}{h_{a}-H_{W}} \delta H_{W} P_{a}+P_{a} \delta H_{W}^{\dagger} \frac{1-P_{a}}{h_{a}-H_{W}} .
$$

The variation of the projectors enters the force always when acting on a vector. To obtain the resulting vector we need to compute the action of an inverse on a vector. If the overlap

Dirac operator is implemented by the rational method of ref [5, 11], the action of $\frac{1}{c+H_{W}}$ on the same vector for some constant $c$ has already been evaluated. Using the shift trick of reference [19] one can evaluate this new inversion at practically no additional cost in operations. 
Of course, if somebody comes up with a replacement of $H_{W}$ that has a larger gap around zero, it just plugs in simply into the overlap alternative.

Projection methods when generalized to domain wall fermions need to address the more complicated form the transfer matrix $T_{W}$. But, if we are willing to depart somewhat from the clean structure we have seen above in the overlap case a natural suggestion is to replace

$H_{W}$ by $H_{W}^{P}$ in the hermitian domain wall operator $H_{\text {dwf }}$. We expect this to help because this would shift the small energy modes of $H_{W}$ elsewhere in the spectrum. This is not an exact procedure in terms of the transfer matrix $T_{W}$, but the unit eigenstates of $T_{W}$ (conjugated by $\gamma_{5}$ ) are also zero eigenstates of $H_{W}$. Viewing the replacement as a perturbation, we see that the troublesome modes are the ones going to be most significantly affected. The main point in choosing an approximate projection is to avoid dealing with the nonlocal structure of the transfer matrix $T_{W}$. Numerical experience from early overlap days teaches us that dealing with $T_{W}$ directly is possible [15], but it is also rewarding to deal with the sparse matrix $H_{W}$ instead. It would be interesting to check this suggestion out in practice.

\section{ADVANTAGES OF THE OVERLAP ALTERNATIVES}

From the point of view of the projection technique the overlap alternative is certainly cleaner. But, if the proposal for domain wall fermions we just made works, we may still feel that the domain wall approach and the overlap approach ended up too close to a draw to make it worthwhile to investigate the overlap alternatives.

Let us now turn to what we feel are more clear advantages of the overlap alternatives. These advantages have to do with the simplified structure of the effective $\psi$ action and with the fact that the light fermion is so well identified. Unlike for domain wall fermions, where the "wave function" for the light quark penetrates a sizable amount into the extra dimension, here the light fermion is fixed as $\psi$. There is no better context to show how this would impact numerical QCD than to discuss chiral symmetry at $\mu=0$ :

Let us introduce new $\psi^{\prime}$ fields so that the action now involves the non-hermitian overlap Dirac operator $D_{o}$ :

$$
\psi^{\prime}=\psi, \quad \bar{\psi}^{\prime}=\bar{\psi} \gamma_{5}
$$

Introduce yet another fermionic degree of freedom $\xi$, but this time an auxiliary one: it has no kinetic energy. Integrating out all the fermions but $\psi$ and $\xi$ leaves the following effective action:

$$
S_{\mathrm{eff}}\left(\psi^{\prime}, \xi\right)=-\bar{\psi}^{\prime} \frac{1+V_{n}}{2} \psi^{\prime}+\bar{\xi} \xi
$$

The matrix $V_{n}$ is given by

$$
V_{n}=\gamma_{5} \varepsilon_{n}\left(H_{W}\right)
$$

and obeys:

$$
V_{n}^{\dagger} V_{n}=\varepsilon_{n}^{2}\left(H_{W}\right)
$$


At $n=\infty$, for an invertible $H_{W}$, we see that $V \equiv V_{\infty}$ is unitary and that the $\bar{\psi}^{\prime}, \psi^{\prime}$ action is given by the overlap Dirac operator, $D_{o}$

$$
D_{o}=\frac{1+V}{2}
$$

It is well known that $D_{o}^{-1}-1$ is chiral [7].

Define the physical fermion field $\psi_{\mathrm{ph}}$ by

$$
\psi_{\mathrm{ph}}=\psi+\xi
$$

This definition was chosen so that we get, at $n=\infty$, the required subtraction of unity (contributed by the $\xi$ propagator) from the $\psi$ propagator.

$$
\left\langle\psi_{\mathrm{ph}} \bar{\psi}_{\mathrm{ph}}\right\rangle=D_{o}^{-1}-1=\frac{1-V}{1+V} .
$$

Therefore, the effective action for the physical field is

$$
S_{\mathrm{eff}}\left(\psi_{\mathrm{ph}}\right)=-\bar{\psi}_{\mathrm{ph}} \frac{1+V}{1-V} \psi_{\mathrm{ph}}
$$

This effective action is chirally symmetric: The transformation

$$
\psi_{\mathrm{ph}} \rightarrow e^{i \alpha \gamma_{5}} \psi_{\mathrm{ph}}, \quad \bar{\psi}_{\mathrm{ph}} \rightarrow \bar{\psi}_{\mathrm{ph}} e^{i \alpha \gamma_{5}}
$$

leaves $S_{\text {eff }}$ invariant on account of

$$
e^{i \alpha \gamma_{5}} \frac{1+V}{1-V} e^{i \alpha \gamma_{5}}=\frac{1+V}{1-V}
$$

A continuum fermion operator with desired chiral properties is now simply transcribed to the lattice by replacing the continuum fermionic fields by $\psi_{\mathrm{ph}}$. Violations of chirality as a result of finite $n$ can be traced quite explicitly.

Perturbative calculations are an integral part of any procedure that connects numerical QCD to physics. In the overlap alternative one can restrict ones attention only to the $\psi$ fields; there is no need to deal with higher dimensional propagators. One has an explicit, relatively simple action, and calculations, although still harder than in the Wilson case, are tractable. Indeed, there has been progress on perturbative calculations with overlap fermions recently [20] (superficially, the treatment of chiral symmetry there may seem somewhat more involved then the one presented above, but it is essentially the same as here). As far as we know there are no finite $n$ calculations yet, but we expect them to be relatively manageable.

The parallel calculations in the domain wall case are extremely cumbersome, see for example [21]. In particular, when one deals with questions related to chiral symmetry one seems to have to deal with the fermions in the five dimensional "bulk" [22]. This makes the finite $n$ effects in the domain wall context harder to estimate quantitatively. All in all, when working with domain wall fermions, one pays quite a price for having the two chirality components of the light fermion widely separated, and the entire set of extra fermions 
actively involved in the communication between them, be it because of finite $n$ effects or be it because of a topologically nontrivial gauge background.

Another factor to recall which is in favor of the overlap has to do with the parameter $m$. In the overlap this parameter is theoretically restricted to the range $-2<m<0$. When the effects of the noisy gauge background are taken into account one gets effectively a positive additive contribution to $m$ (in the mean field approximation it is $d(1-u)$ ) which forces one towards the -2 end of the range. Still, one does not need to go beyond -2 , so one is safe even for trivial gauge backgrounds. In the domain wall case one also is driven towards -2 , but now there is some theoretical worry: For $m$ in the range $-2<m<-1$ the expression for the transfer matrix $T_{W}$, while still a hermitian matrix, no longer stays positive definite for all gauge fields [7]. There are gauge backgrounds for which the matrix has negative eigenvalues. This raises some concerns about the true phase the lattice model is in. Even only the proximity of a phase different from continuum QCD is a source of potentially large, undesirable, numerical effects. Theoretically one would like to stay in the range $-1<m<0$ for domain wall fermions, but this cannot be achieved in QCD at present typical simulation parameters. In principle, one could get into this range, but the needed gauge couplings would have to be impractically weak. Thus, the overlap alternative seems safer for the coarse lattices currently employed in numerical QCD.

Our specific overlap proposal is a very simple implementation of one rational approximation to the sign function. Clearly, there are many variations possible and there is room for more improvement. In terms of flexibility, the overlap alternative of this paper is superior to the domain wall approach; and more flexibility opens more possibilities to increase numerical efficiency.

Up to this point our main conclusion is that the overlap approach based on the matrix $H$ is superior to the one based on domain wall fermions. Of course, we cannot rule out some surprises, so a numerical check in the context of QCD is necessary.

\section{X. $H_{W}$ OPERATION COUNTS}

Going back to our main motivation, the reason to introduce $H$ in the first place was to have a more efficient implementation of the action of the rationally approximated overlap Dirac operator. The original direct implementation used a two level nested conjugate gradient (CG) procedure and a mass-shift trick [5, 11, 19] which makes operation counts almost $n$-independent. In the inner CG the relevant condition number is that of $H_{W}$ and in the outer CG the relevant condition number is roughly $\frac{1}{\mu}$. We see that our worst case bound for the condition number relevant to the single step CG for $H$ is roughly the product of the previous two condition numbers. Thus, it seems that the new procedure is an order $n$ slower than the one it came to improve on. In addition, if one is willing to increase the operation count by a factor of 2 in the old procedure, one can eliminate all the storage of the extra fields, providing a factor of $n$ saving in memory [23]; in a computation limited by memory bandwidth rather than processor performance this version might turn out to be the best.

It is important to mention that these considerations ignore the possibility of preconditioning the algorithm for inverting $H$; for example, the structure of $H$ readily admits standard red-black preconditioning. Other preconditioning methods might exist, exploiting 
the rather smooth structure in $s$-space as evident from the continuum $s$-limit with no $s$ derivatives. Moreover, the bounds on the condition number are not really saturated very often, and super-linear convergence effects in the CG procedure may change the dependence of the number of required iterations on the condition number away from the theoretical limit. Still, it seems that for very large $n$ the older approach will eventually win. However, both the older and newer approach can be improved by projectors, and this will limit the size of $n$ one really needs. Also, the mass-shift trick is incompatible with red-black preconditioning, so this might work in favor of the new approach.

To get some feeling for what one would see in practice we again turn to two dimensional QED, with a simple plaquette Wilson action at $\beta=4$ on an $8 \times 8$ lattice. We performed

the calculations necessary to obtain $\left\langle\bar{\psi}_{\mathrm{ph}} \psi_{\mathrm{ph}}\right\rangle$ using either method and counted the number of $H_{W}$ operations required to reduce the norm of the residual to $10^{-8}$. We used $n=20$ but no preconditioning in the $H$-algorithm. We did use the mass-shift trick in the older algorithm. In neither method did we include projectors. This comparison ended up in favor of the older method by a factor of roughly 2.5. This factor could be beaten by red-black preconditioning, but we have not tried this out.

The above direct comparison between the two overlap methods is easy because they should produce exactly the same results at the same $n$ and $H_{W}$, gauge configuration by gauge configuration. Essentially, these are just two different algorithms to do the same thing. A comparison to domain wall fermions is more difficult, because the differences at finite $n$ are more substantial and there is uncertainty about how to match the parameters. As far as operation counts go, one action of $D$ in the domain wall case counts roughly as $n_{\text {dwf }} H_{W}$ actions.

A plot of the average number of operations of $H_{W}$ as a function of mass is shown for domain wall fermions, for the direct rational implementation and for the higher dimensional implementation of the overlap Dirac operator in Fig. 1. The data was obtained from a sample of 20 configurations. The Wilson mass parameter was set to -1.5 in all cases. Plotted on the vertical axis is the number of operations needed for a single inversion of the fermionic operator. For the overlap Dirac operator simulations $n$ was set to 20 . The plot also includes data for domain wall fermions, where $n$ was taken as 40, in accordance with equation (82). We used the same parameter $\mu$ also for domain wall fermions because the relation in eq. (85) holds only for $0<\mu<<1$ and becomes totally inadequate at $\mu=1$.

\section{CONCLUSIONS}

Let us summarize roughly the situation we were looking at when we began this paper: Although an approach based on the overlap Dirac operator looked theoretically cleaner, domain wall fermions were more attractive numerically. Our analysis has led us to the conclusion that there is no evidence that domain wall fermions have even a numerical advantage.

In all cases we looked at, one faces a problem related to almost zero modes of $H_{W}$. This requires large numbers of extra fields in order to preserve chirality. It also affects adversely the condition numbers. Whichever method we use, the worst case condition numbers are a product of the inverses of two main scale ratios: The first is the scale of the small eigenvalues of $H_{W}^{2}$ divided by an upper bound of the order of 5-10 in lattice units. The second scale 
FIG. 1. A comparison of the number of operations of $H_{W}$ for the inversion of the fermionic operator in three cases: domain wall fermions, the direct implementation of the rational approximated sign function and the higher dimensional implementation of the overlap Dirac operator.

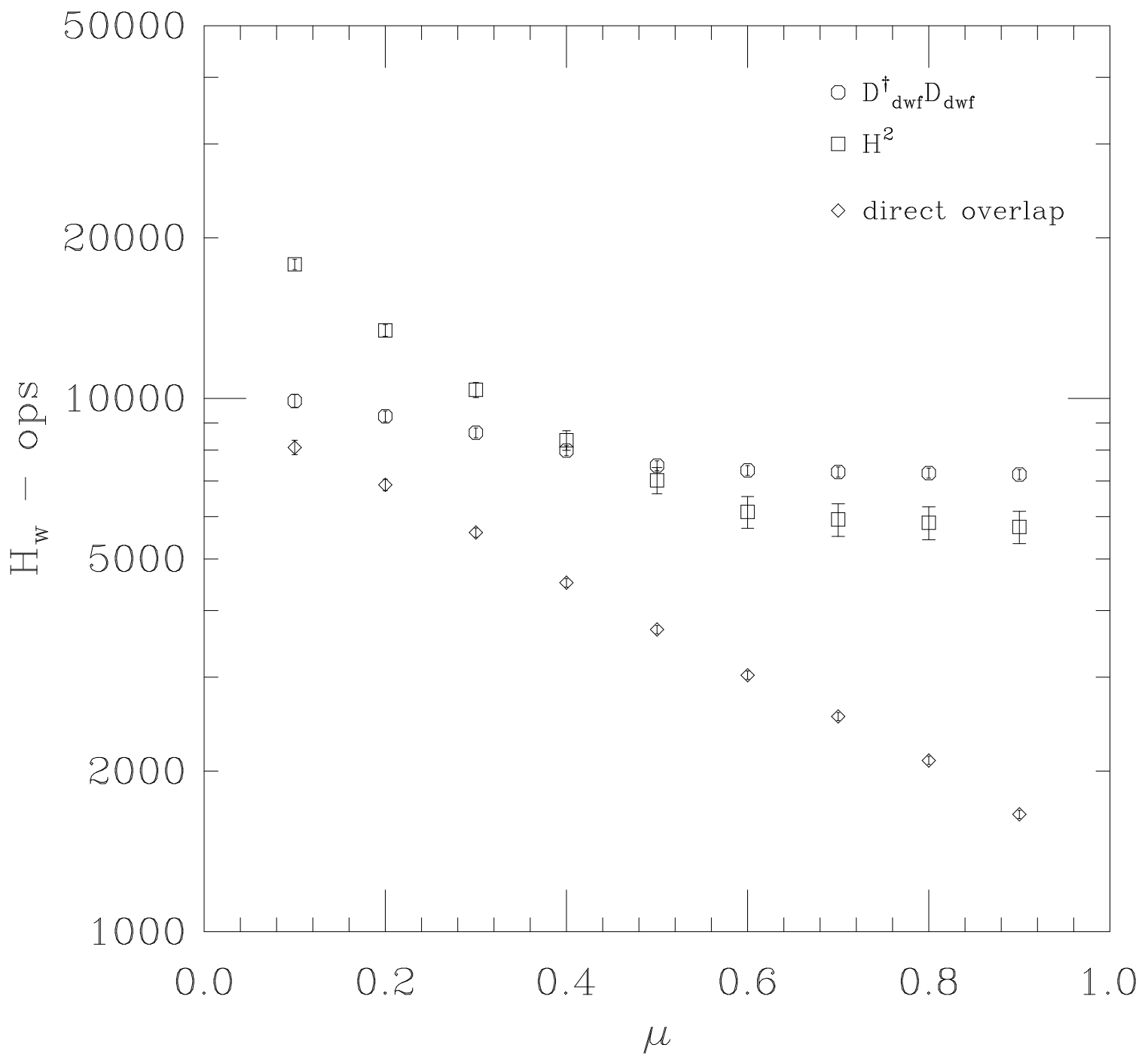


ratio is the lattice physical quark mass squared divided by a number of order unity. Each small scale ratio slows down inversion independently and the effect compounds in the worst case.

Thus, as far as we can see, at the numerical level, there are no a priori advantages to choosing domain wall fermions over overlap fermions in the context of QCD. In both formulations one faces similar numerical obstacles, and the overlap, to say the least, does not fare any worse than domain wall fermions. At the analytical level we are convinced that an approach based on the overlap (or any other efficient replacement of the overlap Dirac operator that might be found in the future) is superior at presently attainable gauge couplings in numerical QCD. Perturbation theory is more transparent to interpret and technically less complex in the overlap version. The chirality violating effects associated with the number of extra fields are much more explicit and therefore their impact should be easier to trace through.

\section{ACKNOWLEDGMENTS}

This research was supported in part by the DOE under grant \# DE-FG05-96ER40559. 


\section{REFERENCES}

[1] D. B. Kaplan, Phys. Lett. B288, 342 (1992).

[2] S. A. Frolov, A. A. Slavnov, Phys. Lett. B309, 344 (1993).

[3] R. Narayanan and H. Neuberger, Phys. Lett. B302, 62 (1993).

[4] D. Boyanovsky, E. Dagotto, and E. Fradkin, Nucl. Phys. B285, 340 (1987); Y. Shamir, Nucl. Phys. B406, 90 (1993).

[5] H. Neuberger, Phys. Rev. Lett. 81, 4060 (1998).

[6] H. Neuberger, Phys. Lett. B417, 141 (1997); Phys. Lett. B427, 125 (1998).

[7] H. Neuberger, Phys. Rev. D57, 5417 (1998).

[8] R.G. Edwards, U.M. Heller, R. Narayanan, hep-lat/9905028; K.F. Liu, S.J. Dong, F.X. Lee, J.B. Zhang, hep-lat/9909061.

[9] P. Vranas, Phys. Rev. D57, 1415 (1998); P. Chen et. al. Nucl. Phys. Proc. Suppl. 73, 456 (1999); T. Blum and A. Soni, Phys. Rev. D56, 174 (1997); J.-F. Lagae, D. K. Sinclair, Nucl. Phys. Proc. Suppl. 73, 450 (1999).

[10] R.G. Edwards, U.M. Heller, R. Narayanan, Nucl. Phys. B535, 403 (1998); Phys. Rev. D60, 034502 (1999).

[11] R. G. Edwards, U. M. Heller, and R. Narayanan, Nucl. Phys. B540, 457 (1998).

[12] R. G. Edwards, U. M. Heller, hep-lat/0005002.

[13] H. Neuberger, Phys. Rev. D60, 065006 (1999).

[14] H. Neuberger, Phys. Rev. D61, 085015 (2000).

[15] R. Narayanan, H. Neuberger, Nucl. Phys. B443, 305 (1995).

[16] G. P. Lepage and P. B. Mackenzie, Phys.Rev. D48, 2250 (1993).

[17] R. G. Edwards, U. M. Heller, and R. Narayanan, Phys. Rev. D59, 094510 (1999).

[18] A. Bode, U. M. Heller, R. G. Edwards, R. Narayanan, hep-lat/9912043.

[19] A. Frommer, S. Güsken, T. Lippert, B. Nöckel, and K. Schilling, Int. J. Mod. Phys. C6, 627 (1995); B. Jergerlehner, hep-lat/9612014.

[20] C. Alexandrou, E. Follana, H. Panagopoulos, E. Vicari, hep-lat/0002010; C. Alexandrou, H. Panagopoulos, E. Vicari, hep-lat/9909158; M. Ishibashi, Y. Kikukawa, T. Noguchi, A. Yamada, hep-lat/9911037.

[21] Y. Kikukawa, H. Neuberger, A. Yamada, Nucl. Phys. B526, 572 (1998).

[22] V. Furman and Y. Shamir, Nucl. Phys. B439, 54 (1995).

[23] H. Neuberger, Int. J. Mod. Phys. C10, 1051 (1999). 